\title{
Role of biofilms in the survival of Legionella pneumophila in a model potable-water system
}

\author{
Ricardo Murga, ${ }^{1}$ Terri S. Forster, ${ }^{1}$ Ellen Brown, ${ }^{2}$ Janet M. Pruckler, ${ }^{2}$ \\ Barry S. Fields ${ }^{2}$ and Rodney M. Donlan ${ }^{1}$ \\ Author for correspondence : Ricardo Murga. Tel: +1 404639 2321. Fax: +1 4046393822. \\ e-mail:rmurga@cdc.gov
}

Epidemiology and Laboratory Branch, Division of Healthcare Quality Promotion 1, and Respiratory Disease Branch, Division of Bacterial and Mycotic Diseases², National Center for Infectious Diseases, Centers for Disease Control and Prevention, Atlanta, GA 30333, USA

\begin{abstract}
Legionellae can infect and multiply intracellularly in both human phagocytic cells and protozoa. Growth of legionellae in the absence of protozoa has been documented only on complex laboratory media. The hypothesis upon which this study was based was that biofilm matrices, known to provide a habitat and a gradient of nutrients, might allow the survival and multiplication of legionellae outside a host cell. This study determined whether Legionella pneumophila can colonize and grow in biofilms with and without an association with Hartmannella vermiformis. The laboratory model used a rotating disc reactor at a retention time of $6.7 \mathrm{~h}$ to grow biofilms on stainless steel coupons. The biofilm was composed of Pseudomonas aeruginosa, Klebsiella pneumoniae and a Flavobacterium sp. The levels of $L$. pneumophila cells present in the biofilm were monitored for $15 \mathrm{~d}$, with and without the presence of $\boldsymbol{H}$. vermiformis, and it was found that, although unable to replicate in the absence of $H$. vermiformis, $L$. pneumophila was able to persist.
\end{abstract}

Keywords : Hartmannella vermiformis, protozoa, growth, ecology

\section{INTRODUCTION}

Legionellae are commonly found in freshwater environments worldwide. However, these bacteria require an unusual combination of nutrients that are rarely found in aquatic environments. When such nutrients are present in the environment, they usually serve to amplify faster-growing bacteria that compete with the legionellae. Legionellae survive as intracellular parasites of free-living protozoa (Fields, 1996; Rowbotham, 1980). Rowbotham (1980) first described the ability of Legionella pneumophila to infect protozoa and later described these bacteria as 'protozoonotic, i.e. naturally infecting protozoa'. Legionellae have been reported to multiply in 13 species of amoebae and two species of ciliated protozoa. Growth of legionellae in the absence of protozoa has been documented only on laboratory media (Fields, 1996). A number of studies have described the relationship between legionellae and protozoa in aquatic environments identified as potential or actual reservoirs of disease-causing strains. Protozoa naturally present in these environments can support intracellular growth of legionellae in vitro (Barbaree et al., 1986; Newsome et al., 1998). In building water systems,

Abbreviation : GFP, green fluorescent protein. microbial growth is frequently detected as biofilms on plumbing fixtures and heating, ventilating and airconditioning equipment. Although it is generally accepted that legionellae are commonly found within these biofilms, most studies have only partially characterized the microbial flora present in the systems (Schofield \& Locci, 1985; Walker et al., 1994). The Legionella biofilm studies that have been conducted employed naturally occurring microbial communities. Several of these studies tested for the presence of protozoan organisms (Fields et al., 1990; Henke \& Seidel, 1986; Surman et al., 1995; Thomas et al., 1999) and found protozoan organisms to be present along with Legionella. An extensive study by Kuchta et al. (1998) examined interactions between L. pneumophila and Hartmannella vermiformis, and the efficacy of several disinfectants, and strongly suggested the requirement of protozoan organisms as a 'growth factor' for the cell replication of Legionella. Some investigators (Wright et al., 1989) have carried out in vitro studies of Legionella biofilm formation and have observed biomass accumulation on the substrata in the absence of protozoan cells. These studies have referred to the biomass as 'growth'; however, they failed to address whether those Legionella cells were actually dividing in the absence of protozoa or merely surviving, which, in our view, is a more relevant question. The purpose of this study was to determine the 
ability of L. pneumophila to grow in a potable-water biofilm without an association with $H$. vermiformis.

\section{METHODS}

Design and assembly of biofilm reactors. Rotating disc reactors (Center for Biofilm Engineering, Bozeman, MT, USA) containing 316L stainless steel coupons $(1.27 \mathrm{~cm}$ in diameter) were used for all experiments and are shown in Fig. 1. The disc reactors were placed in a water bath to hold the temperature at $30^{\circ} \mathrm{C}$. Mixing was provided by a digitally controlled mixing plate (Mirak Thermolyne; Fisher Scientific) placed beneath the water bath. Fig. 1 shows the system set-up diagram. Initially, reactors were operated in batch mode for $72 \mathrm{~h}$ to establish the biofilms on the steel substrata. The medium contained 0.05 g yeast extract, proteose peptone no. 3 , Casamino acids and dextrose, $0.03 \mathrm{~g}$ sodium pyruvate and dibasic potassium phosphate, and $0.005 \mathrm{~g}$ magnesium phosphate per litre of filter-sterilized reverse-osmosis water. Following the period of batch growth, the system was operated as an open system by continuously pumping a $1 / 10$ dilution of the medium formulation given above at a flow rate of $1 \mathrm{ml}$ $\min ^{-1}$ for $24 \mathrm{~h}$ in order to dilute the medium. The feed to the reactors was then changed to filter-sterilized dechlorinated tap water (Atlanta, GA, USA; municipal tap water dechlorinated with $0.5 \mathrm{ml} \mathrm{l}^{-1}$ of a $15.8 \mathrm{~g}$ sodium thiosulfate $\mathrm{l}^{-1}$ solution) at the same flow rate of $1 \mathrm{ml} \mathrm{min}{ }^{-1}$ (retention time of $6.7 \mathrm{~h}$ ). This water had a $\mathrm{pH}$ ranging from $7 \cdot 5$ to $7 \cdot 8$. Each biofilm reactor experiment was repeated at least three times.

Base biofilm bacterial strains. Each reactor was inoculated with Pseudomonas aeruginosa (ATCC 7700), Klebsiella pneumoniae (DMDS Lab. No. 92-08-28a) and a Flavobacterium sp. (CDC-65) organism. These micro-organisms are commonly found in potable-water environments (Geldreich, 1990) and are commonly used in biofilm studies. The strains used in our studies were environmental isolates. Cultures were stored at $-70{ }^{\circ} \mathrm{C}$, transferred to R2A plates (Reasoner \& Geldreich, 1979) and resuspended to a concentration equal to a $0.5 \mathrm{McF}$ arland. Each reactor was inoculated with $1 \mathrm{ml}$ of each cell suspension to a final concentration of approximately $5 \times 10^{5} \mathrm{ml}^{-1}$. Base biofilms were allowed to grow for $7 \mathrm{~d}$ before $H$. vermiformis or $L$. pneumophila was added.

$\boldsymbol{H}$. vermiformis. H. vermiformis (CDC-19) stocks were grown in axenic growth medium at $35^{\circ} \mathrm{C}$ without $\mathrm{CO}_{2}$ (King et al., 1991) and subcultured twice a week into T75 cell-culture flasks. Flasks were tapped on a solid surface to dislodge $H$. vermiformis from the growth surface, transferred to $50 \mathrm{ml}$ conical tubes, centrifuged to pellet the amoebae and

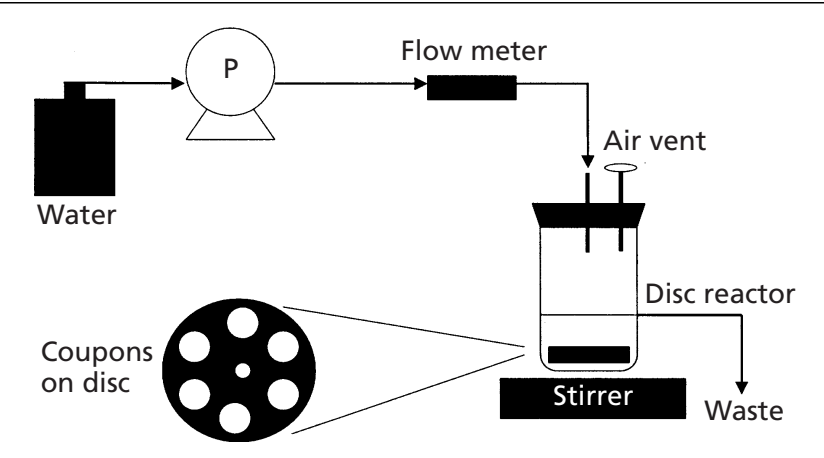

Fig. 1. Potable-water biofilm reactor set-up diagram. resuspended in PBS. Reactors were inoculated with $H$. vermiformis for a final concentration of $10^{4} \mathrm{ml}^{-1}$.

L. pneumophila. L. pneumophila (RI243) carrying the plasmid pANT4 (Lee \& Falkow, 1998) encoding both kanamycin resistance and green fluorescent protein (GFP) was stored as a suspension in defibrinated rabbit blood in a liquid nitrogen $\left(-120^{\circ} \mathrm{C}\right)$ freezer. Fluorescence was determined by using a hand-held lamp [model UVL-21 Blak-Ray Lamp (UVP), longwave UV $333 \mathrm{~nm}$. Four days before the isolate was needed, the mutant was cultured onto BCYE media [buffered charcoalyeast extract agar (containing $0 \cdot 1 \%$ 2-oxoglutarate)] with kanamycin and incubated at $36^{\circ} \mathrm{C}$ with $2.5 \% \mathrm{CO}_{2}$. After the $4 \mathrm{~d}$, the isolate was resuspended in sterile water and diluted to the desired concentration. One millilitre of a suspension of $L$. pneumophila was added to each reactor for a final concentration of approximately $5 \times 10^{5} \mathrm{ml}^{-1}$.

Scanning electron microscopy. Coupons were fixed by placing them into $5 \%$ glutaraldehyde (Ted Pella) in cacodylate buffer (0.067 M, pH 6.2) for fixation overnight at room temperature. Samples were then dehydrated in a graded series of ethanol $(30,50,70,90 \%)$ for $10 \mathrm{~min}$ each at room temperature and immersed in hexamethyldisilazane (Polysciences) for $4 \mathrm{~h}$ at room temperature. Finished specimens were mounted on aluminium stubs with silver paint, sputter-coated with $25 \mathrm{~nm}$ gold, and examined with a Phillips XL 30 environmental scanning electron microscope (FEI, a subsidiary of Phillips).

Epifluorescence microscopy. Coupons were fixed by placing them into $5 \%$ formaldehyde (J. T. Baker) in reverse osmosis water for $5 \mathrm{~min}$ at room temperature. Samples were then fluorescently stained with $1 \mu \mathrm{g} \mathrm{4}$, 6 -diamidino-2-phenylindole (Sigma) $\mathrm{ml}^{-1}$ for $15 \mathrm{~min}$; this was followed by rinsing in reverse osmosis filter-sterilized water. The coupon surfaces were examined with an Axioskop 2 epifluorescence microscope (Carl Zeiss) using an HBO-100 illuminator and a Zeiss Plan-NEOFLUAR $\times 1001.30$ oil objective with a $355 / 40$ excitation filter, a 400/long-pass dichroic mirror and a $420 /$ long-pass emission filter. To visualize the GFP cells, we examined the surfaces with a 480/40 excitation filter, a 505 dichroic mirror and a 510/long-pass emission filter.

Processing for the removal of biofilms. Coupons were removed from the reactors, dip-rinsed in phosphate-buffer water, placed into $10 \mathrm{ml}$ phosphate-buffer saline, processed by three cycles of sonication for $30 \mathrm{~s}$ followed by vortexing for $30 \mathrm{~s}$, homogenized for $1 \mathrm{~min}$, and spread-plated on R2A medium for quantification of the base biofilm. For the recovery of $H$. vermiformis, $100 \mu \mathrm{l}$ aliquots from several dilutions were plated onto non-nutritive agar that had been spread with viable Escherichia coli. Plates were read at 3 and $7 \mathrm{~d}$ for the presence/absence of H.vermiformis at the dilution plated. For the recovery of L. pneumophila, the supernate from the processing of each coupon was treated with a $\mathrm{KCl} / \mathrm{HCl}$ solution, filtered through a $0.2 \mu \mathrm{m}$ filter (part no. GTTP; Millipore), resuspended, and plated onto glycine-polymixin B-anisomycin-vancomycin plates.

\section{RESULTS}

Base biofilm densities of $10^{7}-10^{8}$ c.f.u. per coupon were consistently recovered from disc reactors prior to the inoculation of the system with $H$. vermiformis. Fig. 2 shows the effect of the addition of $H$. vermiformis on the base biofilm. Base biofilm counts decreased by approximately 2 logs, and biofilm-associated $H$. vermiformis increased by approximately $2 \operatorname{logs}$ between day 8 and day 10; these levels were maintained for the remainder 


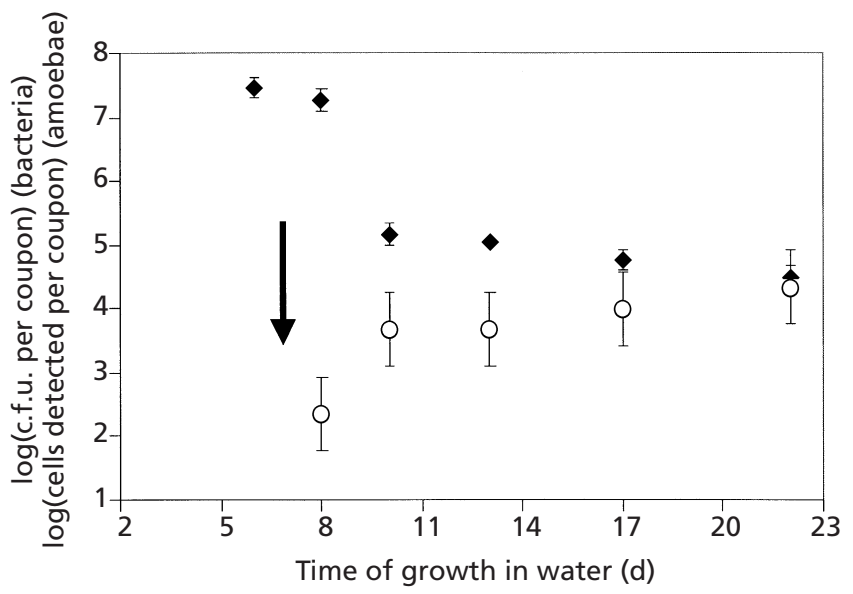

Fig. 2. Recovery of biofilm-associated $H$. vermiformis from a potable-water biofilm. The arrow indicates the day of inoculation with $H$. vermiformis. $\diamond, P$. aeruginosa, $K$. pneumoniae and a Flavobacterium sp. in the biofilm. $O, H$. vermiformis in the biofilm. Error bars indicate the SD $(n=3)$.
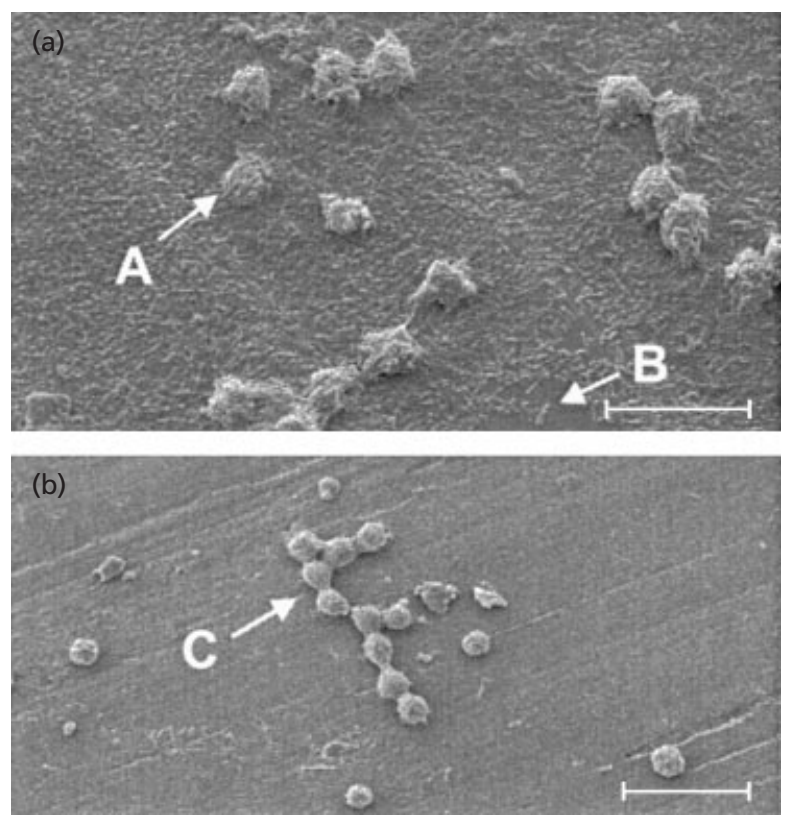

Fig. 3. Scanning electron micrographs of the biofilms $1 \mathrm{~d}$ (a) and $15 \mathrm{~d}(\mathrm{~b})$ after the addition of $H$. vermiformis. A, B and $C$, $H$. vermiformis trophozoites covered with bacteria, bacterial cells, and $H$. vermiformis cysts, respectively. Bars, $20 \mu \mathrm{m}$.

of the experiment (a total of $15 \mathrm{~d}$ ). The reduction in the base biofilm levels was apparently due to predation by $H$. vermiformis, since the biofilm-associated $H$. vermiformis growth rates correlated with the base biofilm rate of decline, and no such rapid decline in base biofilm occurred in the absence of H. vermiformis (see Fig. 5).

Scanning electron micrographs of $H$. vermiformis on the base biofilm, 1 and $15 \mathrm{~d}$ after addition of the amoebae, respectively, are shown in Fig. 3(a, b). H. vermiformis

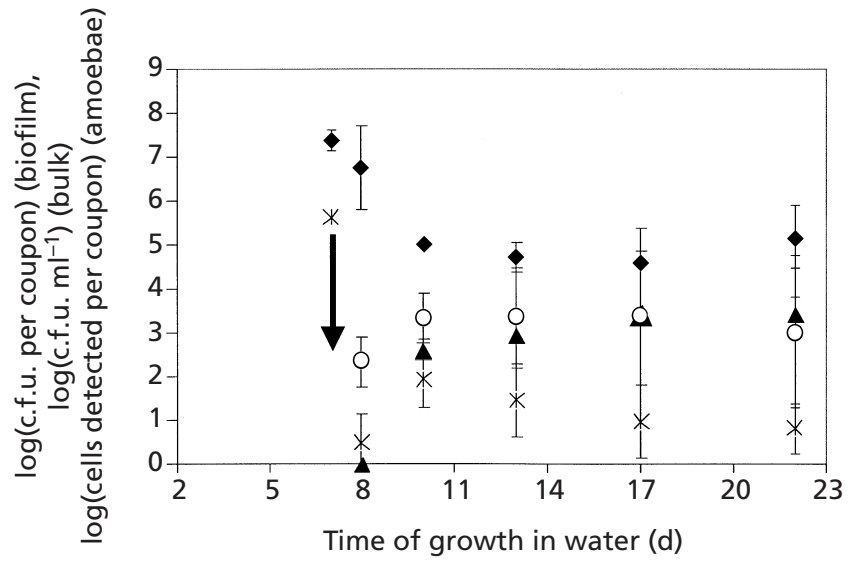

Fig. 4. Recovery of biofilm-associated $H$. vermiformis and $L$. pneumophila from a potable-water biofilm. The arrow indicates the day of inoculation with $H$. vermiformis and $L$. pneumophila. $\diamond, P$. aeruginosa, $K$. pneumoniae and a Flavobacterium sp. in the biofilm. $\boldsymbol{\Delta}, L$. pneumophila in the biofilm. $\bigcirc, H$. vermiformis in the biofilm. $\times, L$. pneumophila in bulk liquid. Error bars indicate the SD $(n=3)$.

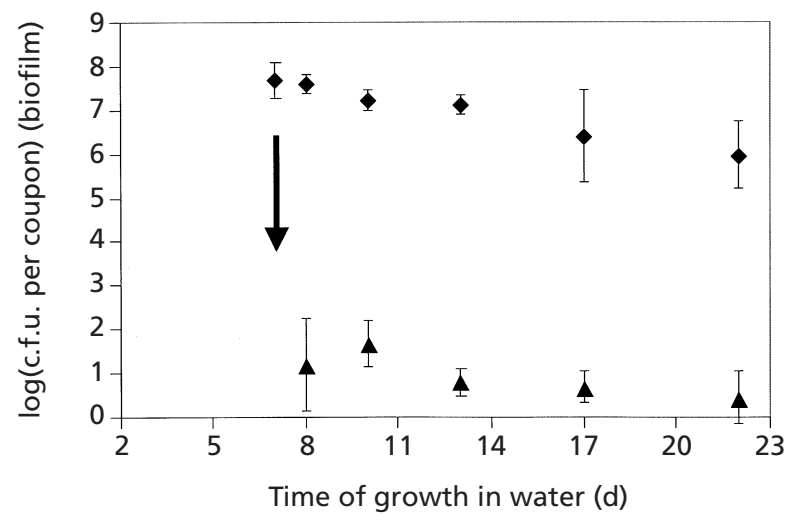

Fig. 5. Recovery of biofilm-associated L. pneumophila from a potable-water biofilm. The arrow indicates the day of inoculation with $L$. pneumophila. $\diamond, P$. aeruginosa, $K$. pneumoniae and a Flavobacterium sp. in the biofilm. $\boldsymbol{\Delta}$, L. pneumophila in the biofilm. Error bars indicate the SD $(n=3)$.

trophozoites (Fig. 3a) encyst as they feed on the base biofilm and reduce it (Fig. 3b). H. vermiformis encysted as early as $6 \mathrm{~d}$ after their addition. When L. pneumophila and H. vermiformis were added to the reactor on day 7 , a similar reduction in the base biofilm occurred, whereas the $H$. vermiformis and $L$. pneumophila counts increased (Fig. 4). L. pneumophila counts in the biofilm reached $10^{3}$ c.f.u. per coupon, and remained above $10^{3}$ for the duration of the experiment. Addition of $L$. pneumophila to the base biofilm without $H$. vermiformis resulted in much lower numbers of L. pneumophila (1-2 logs fewer) being recovered from the biofilms (Fig. 5). In addition, a comparison between Fig. 4 and Fig. 5 shows that planktonic L. pneumophila cells were recovered only from the disc reactors inoculated with both $H$. 
Table 1. Validation of plasmid loss as a marker of growth in a batch study

Legionella recovered are expressed as mean c.f.u. $\mathrm{ml}^{-1}$ for the bulk $(n=3)$. The percentage GFP is presented in parentheses.

\begin{tabular}{|lll|}
\hline $\begin{array}{l}\text { Time after } \\
\text { inoculation }(d)\end{array}$ & With amoebae & Without amoebae \\
\hline 0 & $1 \cdot 1 \times 10^{3}(99 \cdot 3)$ & $1 \cdot 6 \times 10^{3}(99 \cdot 2)$ \\
3 & $8 \cdot 9 \times 10^{6}(74 \cdot 2)$ & $1 \cdot 6 \times 10^{3}(99 \cdot 3)$ \\
7 & $3 \cdot 0 \times 10^{8}(77 \cdot 7)$ & $1 \cdot 0 \times 10^{2}(93 \cdot 7)$ \\
\hline
\end{tabular}

vermiformis and L. pneumophila. These results appeared to support the conclusions that $H$. vermiformis colonized the base biofilms, and that the ability of $L$. pneumophila to colonize this system was significantly improved when $H$. vermiformis was present.

To determine if L. pneumophila was multiplying in the absence of the amoebae, we looked at the rate of plasmid loss by L. pneumophila in biofilms with and without $H$. vermiformis. Loss of this plasmid can readily be determined by loss of fluorescence. Loss of the plasmid was confirmed in the nonfluorescing bacteria by loss of the ability to grow on kanamycin. To ensure that L. pneumophila loses fluorescence when multiplying, the bacteria were coinoculated with $H$. vermiformis into assay medium, and loss of fluorescence was determined over $7 \mathrm{~d}$ (Table 1, column 2). To determine the loss of fluorescence from L. pneumophila cells that were not in the process of multiplying, we suspended L. pneumophila cells in sterile water without $H$. vermiformis, and the loss of fluorescence was determined during the $7 \mathrm{~d}$ (Table 1, column 3). These results indicated that $L$. pneumophila numbers increased exponentially when associated with the amoebae, and that the percentage of fluorescent cells decreased steadily over the $7 \mathrm{~d}$. The percentage of cells fluorescing (and total viable counts) without amoebae (column 3) was essentially unchanged when cells were suspended in sterile water. For both datasets, the percentage of cells carrying the plasmid is shown in parentheses.

The percentage of fluorescing cells in biofilm-associated L. pneumophila in the biofilm reactor study is shown in Table 2. The percentage of fluorescing cells in the biofilms ranged from 82 to $89 \%$ between 1 and $15 \mathrm{~d}$ after inoculation with $H$. vermiformis and L. pneumophila (Table 2, column 3). The percentage of fluorescing planktonic L. pneumophila cells ranged from 71 to $100 \%$ (column 2) and, as with the biofilm cells, decreased over time. Biofilm-associated L. pneumophila in the absence of $H$. vermiformis showed an increase from $98 \% 1 \mathrm{~d}$ after the addition of L. pneumophila to $99 \%$ on day 2, and to $100 \%$ fluorescing cells for the remainder of the experiment (column 5). No planktonic L. pneumophila was detected [the limit of detection was 1.3 c.f.u. (ml bulk liquid) ${ }^{-1}$ ] in the reactor without $H$. vermiformis. All isolated fluorescent colonies maintained their fluorescence upon repeated transfer to fresh medium and grew in the presence of kanamycin (a measurement of plasmid-encoded kanamycin resistance). Isolates that had lost their fluorescence failed to grow in the presence of kanamycin.

Microscopic observations of the biofilm matrix showed no evidence of microcolony formation by GFP cells in either the presence or the absence of H. vermiformis.

Our results (Figs 4 and 5, Tables 1 and 2) showed that, in our biofilm system, H. vermiformis was required for L. pneumophila multiplication, and that the biofilm matrix in the absence of $H$. vermiformis provided an environment in which $L$. pneumophila could survive without division.

\section{DISCUSSION}

Cell growth can be defined as the culmination of an orderly interplay between all the physiological activities of the cell. It is a complex process involving the uptake of nutrients, conversion of these nutrients into usable energy, replication of the chromosome, increases in the size and mass of the cell, and division into two daughter

Table 2. Assessment of Legionella growth, in a biofilm study, based upon GFP plasmid loss

Legionella recovered are expressed as mean c.f.u. per coupon for the biofilm $(n=3)$. The percentage GFP is presented in parentheses. ND, Not detected.

\begin{tabular}{|c|c|c|c|c|}
\hline \multirow{2}{*}{$\begin{array}{l}\text { Time after } \\
\text { inoculation }(d)\end{array}$} & \multicolumn{2}{|c|}{ With amoebae } & \multicolumn{2}{|c|}{ Without amoebae } \\
\hline & Bulk & Biofilm & Bulk & Biofilm \\
\hline 0 & - & - & ND & - \\
\hline 1 & $4 \cdot 6 \times 10^{0}(100 \cdot 0)$ & - & ND & $4 \cdot 1 \times 10^{1}(98 \cdot 5)$ \\
\hline 3 & $1 \cdot 7 \times 10^{2}(91 \cdot 8)$ & $4 \cdot 5 \times 10^{2}(88 \cdot 6)$ & ND & $7 \cdot 8 \times 10^{1}(99 \cdot 2)$ \\
\hline 6 & $8 \cdot 3 \times 10^{1}(81 \cdot 7)$ & $1 \cdot 2 \times 10^{3}(86 \cdot 3)$ & ND & $7 \cdot 6(100 \cdot 0)$ \\
\hline 10 & $1 \cdot 8 \times 10^{1}(78 \cdot 9)$ & $5 \cdot 5 \times 10^{3}(87 \cdot 8)$ & $\mathrm{ND}$ & $6 \cdot 2(100 \cdot 0)$ \\
\hline 15 & $1 \cdot 0 \times 10^{1}(71 \cdot 4)$ & $3 \cdot 6 \times 10^{3}(82 \cdot 1)$ & ND & $4.9(100 \cdot 0)$ \\
\hline
\end{tabular}


cells (Moat \& Foster, 1995). According to this definition, active cell division and the production of daughter cells are among the prerequisites for growth. Terms such as 'viable but nonculturable' have been used by other investigators to describe cells that might be injured and, though metabolically active, incapable of cell division. Steinert et al. (1997) determined the recovery of $L$. pneumophila suspended in sterile water for $180 \mathrm{~d}$ by using routine plating media and acridine orange direct counts. They found that viable plate counts declined steadily during the first $125 \mathrm{~d}$ incubation, after which the cells were no longer culturable. However, the rate of decline in acridine orange direct counts was much smaller, and cells were detected even after $55 \mathrm{~d}$ nonculturability. When Acanthamoeba castellanii was added to their system containing only nonculturable cells, culturable cells of L. pneumophila were detected after $1 \mathrm{~d}$ coincubation. Multiplication of L. pneumophila was evidenced by the fact that cell concentrations exceeded day 1 concentrations $3 \mathrm{~d}$ after inoculation with A. castellanii. Their results strongly support the findings of Kuchta et al. (1998), mentioned earlier. Because we were able to culture L. pneumophila cells from biofilms, it was apparent that at least a percentage of cells in our system were both viable and culturable. However, it was still unclear as to whether L. pneumophila cells recovered from the biofilms in the absence of amoebae were actually growing (in every sense of the definition given earlier) or simply surviving by endogenous metabolism in the early stages of the 'viable but nonculturable' state. To address this question, we used plasmid loss as an indication of cell division of L. pneumophila. The strain of L. pneumophila used carried a plasmid encoding both kanamycin resistance and GFP. A lack of fluorescence was interpreted as evidence of plasmid loss, which would serve to indicate cell division in the absence of selective pressure. After L. pneumophila + GFP were inoculated into a medium containing $H$. vermiformis (but without a biofilm), the L. pneumophila cell count increased exponentially with a steady loss of fluorescence. L. pneumophila suspended in water without $H$. vermiformis (and without a biofilm) showed no such growth or loss of fluorescence. Studies in the biofilm reactors confirmed the batch studies; virtually all of the $L$. pneumophila cells in biofilms without $H$. vermiformis maintained fluorescence, whereas those with $H$. vermiformis in the biofilms lost fluorescence and produced increased cell counts. These results, combined with the fact that microcolony formation was never observed, argue that although the presence of $H$. vermiformis is not required for survival, it is required for growth in our model system. Similar findings were reported at a recent international meeting (Szewzyk et al., 2000). Using a continuous flow chamber described elsewhere (Szewzyk et al., 1994), the investigators followed the behaviour of a L. pneumophila serogroup 1 strain (LP1) in the biofilm and the outflow water over $98 \mathrm{~d}$. Their findings showed that L. pneumophila did not multiply in defined mixed biofilms with a natural water bacterium. The number of LP1 cells decreased rapidly in the outflowing water of the mixed biofilm, and no LP1 cells were detected in the biofilm after $40 \mathrm{~d}$ (by fluorescent in situ hybridization using a Legionella-specific probe). In a parallel chamber A. castellanii (ATCC 33152) was added simultaneously to LP1. The number of LP1 cells in the outflowing water increased to several logs $\mathrm{ml}^{-1}$ and remained constant for $98 \mathrm{~d}$. Colonized amoebae were detected in the biofilm by fluorescent in situ hybridization with probes specific to eukaryotes and Legionella. Only half of the amoebae detected contained LP1 cells, and only $10 \%$ were heavily colonized. Some Legionella cells were detected outside the amoebae but were always in close proximity to the amoebae.

Our model system does not exactly replicate conditions provided in natural potable-water biofilms, and a different set of conditions could provide the necessary growth requirements for Legionella without associated free-living protozoa. However, the significant differences between Hartmannella-containing and nonHartmannella-containing biofilms in terms of the Legionella growth rate, plasmid loss and production of daughter cells indicate that H. vermiformis (and probably other free-living protozoa) plays a role in the survival and growth of Legionella in the environment.

\section{Conclusions}

A biofilm reactor capable of developing reproducible, steady-state bacterial biofilms in nonsupplemented potable water has been developed. We chose this model because we believe that it provides a realistic representation of the conditions and organisms that we wanted to study.

H. vermiformis was shown to associate with these biofilms and feed upon the bacteria within them. Under our system's conditions, viable L. pneumophila associated and persisted in these biofilms with and without $H$. vermiformis for a period of $15 \mathrm{~d}$ after inoculation.

L. pneumophila cells did not develop microcolonies or biofilms in the biofilm reactors, even in the presence of $H$. vermiformis. This suggests that their presence in our biofilms could be attributed to the survival of cells from the original inoculum in the biofilm matrix, plus daughter cells that resulted from cell division inside a protozoan host.

In this biofilm reactor system, higher numbers of $L$. pneumophila were recovered from the biofilm matrix in the presence of $H$. vermiformis. Also, planktonic $L$. pneumophila cells were detected only in the bulk liquid from reactors that contained $H$. vermiformis. The quantification of the GFP loss by L. pneumophila was used as an indication of cell replication, and demonstrated that replication occurred only in the presence of H. vermiformis.

On the basis of this and other similar studies, we conclude that although L. pneumophila was not capable of growth in the absence of the protozoan host, this organism could survive within the biofilm matrix. In 
agreement with the current understanding of biofilms, we demonstrated that the presence of biofilms in potable and healthcare-facility water systems can provide a means for the survival and dissemination of L. pneumophila.

\section{ACKNOWLEDGEMENTS}

We thank Janice Carr for the scanning electron microscopy of our biofilms. Use of trade names and commercial sources is for identification only and does not imply endorsement by the PHS of the USDHHS.

\section{REFERENCES}

Barbaree, J. M., Fields, B. S., Feeley, J. C., Gorman, G. W. \& Martin, W. T. (1986). Isolation of protozoa from water associated with a legionellosis outbreak and demonstration of intracellular multiplication of Legionella pneumophila. Appl Environ Microbiol 51, 422-424.

Fields, B. S. (1996). The molecular ecology of legionellae. Trends Microbiol 4, 286-290.

Fields, B. S., Nerad, T. A., Sawyer, T. K., King, C. H., Barbaree, J. M., Martin, W. T., Morril, W. E. \& Sanden, G. N. (1990). Characterization of an axenic strain of Hartmanella vermiformis obtained from an investigation of nosocomial legionellosis. $J$ Protozool 37, 581-583.

Geldreich, E. E. (1990). Microbiological quality control in distribution systems. In Water Quality and Treatment: a Handbook of Community Water Supplies, pp. 1113-1158. Edited by F. W. Pontius, American Water Works Association. New York: McGraw-Hill.

Henke, M. \& Seidel, K. M. (1986). Association between Legionella pneumophila and amoebae in water. Isr J Med Sci 22, 690-695.

King, C. H., Fields, B. S., Shotts, E. B., Jr \& White, E. H. (1991). Effects of cytochalasin D and methylamine on the intracellular growth of Legionella pneumophila in amoebae and human monocyte-like cells. Infect Immun 59, 758-763.

Kuchta, J. M., States, S. J., Wadowsky, R. M. \& Byers, T. J. (1998). Interactions of Legionella pneumophila with Hartmannella vermiformis including the efficacy of chlorine or copper and silver ions to disrupt the intra-amoebic multiplication of L. pneumophila. Recent Res Dev Microbiol 2, 405-425.

Lee, A. K. \& Falkow, S. (1998). Constitutive and inducible green fluorescent protein expression in Bartonella henselae. Infect Immun 66, 3964-3967.
Moat, A. G. \& Foster, J. W. (1995). Microbial Physiology, 3rd edn, pp. 19-20. New York: Wiley-Liss.

Newsome, A. L., Scott, T. M., Benson, R. F. \& Fields, B. S. (1998). Isolation of an amoeba naturally harboring a distinctive Legionella species. Appl Environ Microbiol 64, 1688-1693.

Reasoner, D. J. \& Geldreich, E. E. (1979). A new medium for enumeration and subculture of bacteria from potable water. Appl Environ Microbiol 49, 1-7.

Rowbotham, T. J. (1980). Preliminary report on the pathogenicity of Legionella pneumophila for freshwater and soil amoebae. J Clin Pathol 33, 1179-1183.

Schofield, G. M. \& Locci, R. (1985). Colonization of components of a model hot water system by Legionella pneumophila. J Appl Bacteriol 58, 151-162.

Steinert, M., Emody, L., Amann, R. \& Hacker, J. (1997). Resuscitation of viable but nonculturable Legionella pneumophila Philadelphia JR32 by Acanthamoeba castellanii. Appl Environ Microbiol 63, 2047-2053.

Surman, S. B., Morton, L. H. G. \& Keevil, C. W. (1995). The use of a biofilm generator in the evaluation of a biocide for use in water treatment. In Proceedings of the 9th International Biodeterioration and Biodegradation Symposium, 5-10 September 1993, Leeds, UK, pp. 7-16. Edited by A. Bousher, M. Chandra \& R. Edyvean. Rugby: Institute of Chemical Engineers.

Szewzyk, R., Bäz, G. \& Bartocha, W. (2000). Survival and growth of Legionella pneumophila in biofilms of drinking water bacteria and amoebae. In American Society for Microbiology Conference on Biofilms 2000, Big Sky, MT, USA, poster no. 145. Washington, DC: American Society for Microbiology.

Szewzyk, U., Manz, W., Amann, R., Schleifer, K. H. \& Stenstrom, T. A. (1994). Growth and in-situ detection of a pathogenic Escherichia coli in biofilms of a heterotrophic water-bacterium by use of 16S-ribosomal-RNA-directed and 23S-ribosomal-RNAdirected fluorescent oligonucleotide probes. FEMS Microbiol Ecol 13, 169-175.

Thomas, W. M., Eccless, J. \& Fricker, C. (1999). Laboratory observations of biocide efficacy against Legionella in model cooling tower system. ASRAE Trans 105, 607-623.

Walker, J. T., Rogers, J. \& Keevil, C. W. (1994). An investigation of the efficacy of bromine containing biocide on an aquatic consortium of planktonic and biofilm micro-organisms including Legionella pneumophila. Biofouling 8, 47-54.

Wright, J. B., Ruseska, I., Athar, M. A., Corbett, S. \& Costerton, J. W. (1989). Legionella pneumophila grows adherent to surfaces in vitro and in situ. Infect Control Hosp Epidemiol 10, 408-415.

Received 18 May 2001 ; revised 16 July 2001; accepted 27 July 2001. 